\title{
Mammalian Target of Rapamycin as a Rational Therapeutic Target for Breast Cancer Treatment
}

\author{
Patricia Mucci LoRusso \\ Karmanos Cancer Institute/Wayne State University, Detroit, Mich., USA
}

\section{Key Words}

Mammalian target of rapamycin $\cdot \mathrm{mTOR} \cdot$ Estrogen

receptor $\cdot$ Human epidermal growth factor receptor 2 inhibitors from laboratory studies to large clinical trials, driven by a rational understanding of the role of mTOR in the processes that underlie breast cancer tumorigenesis.

Copyright $\odot 2012$ S. Karger AG, Basel

\section{Introduction}

Breast cancer, accounting for $28 \%$ of all cancers affecting women in the United States, is the most common cancer among women, with over 207,000 new cases reported in 2010 [1]. Even though death rates have fallen steadily since 1990, reflecting improvements in early detection and treatment, mortality remains high, with breast cancer being the second most common cause of cancerrelated deaths in women [1]. These high death rates reflect the limited effectiveness of current therapeutic options, particularly in patients with advanced disease.

Treatment decisions are guided by stage, tumor grade and hormone and human epidermal growth factor receptor 2 (HER2) status. Targeted therapies, such as endocrine and biological therapy (e.g. trastuzumab), were developed based on rational understanding of processes underlying tumorigenesis resulting in significant improvements in treatment outcomes [2]. However, similar to traditional chemotherapy [3], drug resistance with

\section{KARGER}

Fax +41613061234 E-Mail karger@karger.ch www.karger.com
(ㄷ) 2012 S. Karger AG, Basel

0030-2414/13/0841-0043\$38.00/0

Accessible online at:

www.karger.com/ocl
Patricia M. LoRusso, DO

Center for Experimental Therapeutics, Karmanos Cancer Institute

4100 John R, HWCRC Rm 4206/Mailcode: HW04HO

Detroit, MI 48201 (USA)

E-Maillorussop@karmanos.org 
Fig. 1. $\mathrm{PI} 3 \mathrm{~K} / \mathrm{mTOR}$ pathway [5]. 4EBP1 = 4E-binding protein 1; AMPK = adenosine monophosphate-activated protein kinase; ASK1 = apoptosis signal-regulating kinase 1 ; ATP = adenosine- 5 '-triphosphate; $\mathrm{BAD}=\mathrm{BCL} 2$-associated agonist of cell death; eEF2K = eukaryotic elongation factor-2 kinase; eIF4B = eukaryotic initiation factor $4 \mathrm{~B}$; eIF4E = eukaryotic initiation factor $4 \mathrm{E}$; FKBP12 = FK506-binding protein, $12 \mathrm{kDa}$; FOXO = forkhead box O1; GDP = guanosine diphosphate; GSK3 = glycogen synthase kinase 3; GTP = guanosine-5'triphosphate; IRS1 = insulin receptor substrate $1 ;$ mLST8 = mTOR associated protein, LST8 homolog; $\mathrm{mTOR}=$ mammalian target of rapamycin; $\mathrm{mTORC1}=$ mTOR complex $1 ;$ mTORC $2=$ mTOR complex 2; PDCD4 = programmed cell death 4; $\mathrm{PDK} 1$ = phosphoinositide-dependent kinase $1 ; \mathrm{PI} 3 \mathrm{~K}=$ phosphatidylinositol 3-kinase; PIP2 = phosphatidylinositol $(4,5)$ biphosphate; PIP3 = phosphatidylinositol $(3,4,5)$ triphosphate; PRAS40 = prolinerich Akt substrate 40; $\mathrm{PTEN}=$ phosphatase and tensin homolog; Rheb = Ras homolog enriched in brain; S6 = ribosomal protein S6; S6K = ribosomal protein S6 kinase; SIN1 = stress-activated mitogen-activated protein kinase associated protein 1 ; $\mathrm{TSC} 1=$ tuberous sclerosis complex 1; TSC2 = tuberous sclerosis complex 2. Reprinted from [5]. Copyright (C) 2010 with permission from Elsevier.

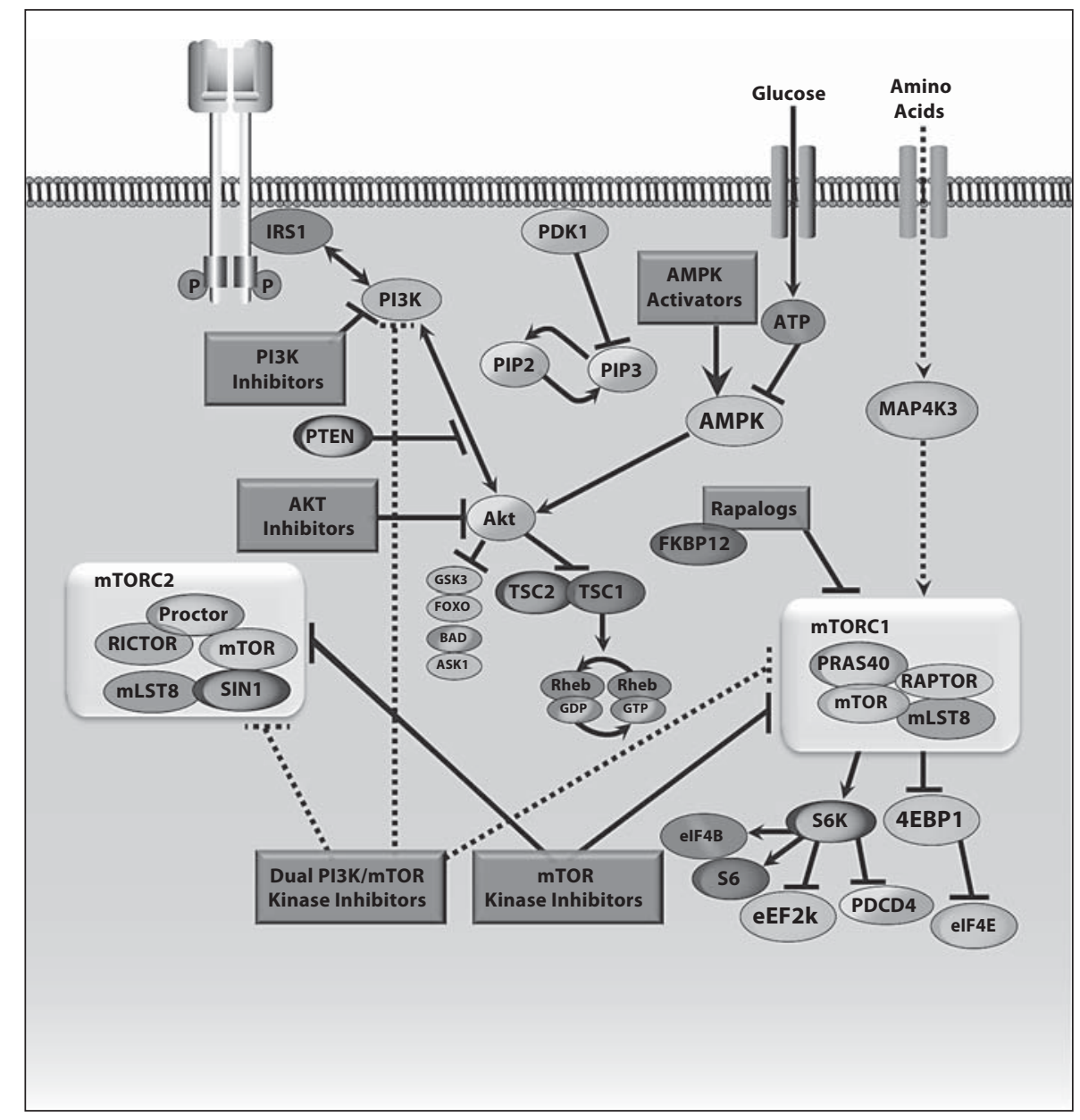

these therapies still represents a significant problem resulting in disease recurrence and/or progression, necessitating alternative treatment strategies.

The mammalian target of rapamycin (mTOR), a ubiquitously expressed protein kinase and important regulator of cell growth and proliferation, is implicated in cell processes that lead to uncontrolled growth of cancer cells. mTOR inhibitors are effective against several cancer types, and emerging data support their use in breast cancer. A number of clinical candidates have undergone, or are undergoing, evaluation against this tumor type, yielding interesting data implicating a pivotal role for the mTOR pathway in this disease.

\section{Overview of the mTOR Pathway}

mTOR is a serine-threonine protein kinase that plays an integral role in signal transduction pathways that control cell growth and survival [4]. This cellular mediator is critical in translating the effects of growth factors and nutrients at the cell surface into appropriate adaptive responses involving protein translation and transcription. mTOR consists of 2 protein complexes: mTOR complexes 1 and 2 (mTORC1 and mTORC2). mTORC1 is regulated by a number of signaling pathways, including the phosphatidylinositol-3-kinase (PI3K)/protein kinase B (Akt) pathway (fig. 1) [5]. Activation of PI3K by growth factors and nutrients, with subsequent activation of Akt, has 2 notable effects: first, it prevents dimerization of tuberous sclerosis complexes (TSCs) 1 and 2, and second, it promotes phosphorylation of proline-rich Akt substrate 40 $\mathrm{kDa}$ (PRAS40). Both inhibition of TSC dimerization and phosphorylation of PRAS40 allow mTORC1 to promote cell growth and proliferation via downstream transduction of proliferative signals through phosphorylation of the translational regulator eukaryotic initiation factor $4 \mathrm{E}$-binding protein (4E-BP1) and the ribosomal protein S6 kinase [6]. In contrast, under conditions of depleted energy stores, TSC2 activity increases, causing downreg- 
Fig. 2. Cross-talk between signal transduction pathways and ER signaling in endocrine-resistant breast cancer. Republished with permission of American Association for Cancer Research from [8]. Copyright (C) 2010; permission conveyed through Copyright Clearance Center, Inc.

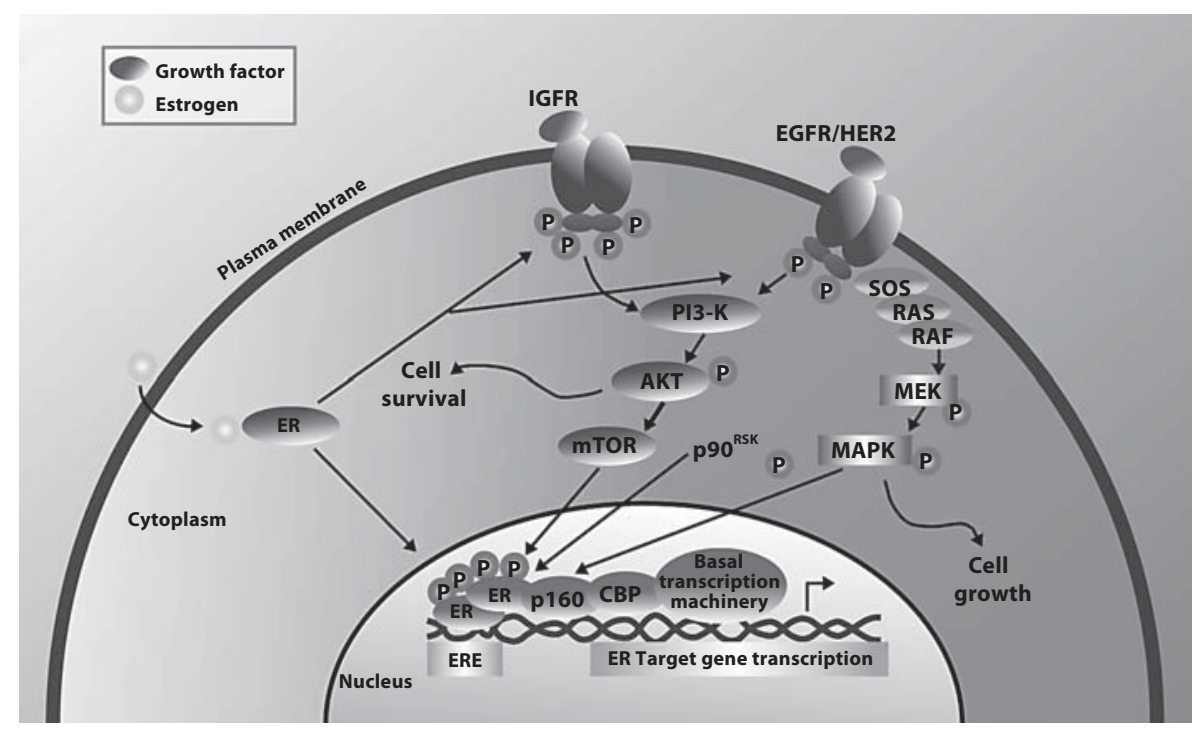

ulation of mTORC1 and a consequent reduction in growth. mTORC1 is the biological target for rapamycin and other mTOR inhibitors, which are thought to exert their effects by destabilizing the structural integrity of mTORC1, weakening the association of MTOR with its regulatory associated protein raptor, and blocking downstream interactions with S6 kinase [7]. mTORC2 is also a multiprotein complex that has $\geq 2$ major functions: regulation and organization of the cellular actin cytoskeleton and regulation of Akt through phosphorylation. For full activation, Akt requires phosphorylation by both mTORC2 and PI3K [4].

One of the key regulators of the PI3K/Akt/mTOR pathway is the phosphatase tensin homolog (PTEN) [4]. Under normal conditions, PTEN acts as a negative regulator of this pathway, limiting the growth proliferationpromoting effects mediated through activation of mTORC1. Mutations or gene amplifications in PI3K and mutations resulting in PTEN loss are reported in many cancers, making the mTOR signaling pathway a rational therapeutic target for cancer drugs [4].

\section{Rationale for Using mTOR Inhibitors in Breast Cancer}

Although endocrine therapy and HER2-targeted therapy are considered effective, resistance occurs limiting further treatment options. However, an accumulating body of evidence indicates that overactivation of the $\mathrm{PI} 3 \mathrm{~K} / \mathrm{Akt} / \mathrm{mTOR}$ pathway may represent a key process in the development of resistance to these treatments (fig. 2) [8]. It has been postulated that the use of mTOR inhibitors in breast cancer may be twofold: in patients with acquired resistance, they could be used to partially restore sensitivity and provide an additional treatment period for drugs such as trastuzumab or aromatase inhibitors, whereas in patients who have not previously been treated with endocrine therapies, they could be used in combination with hormonal treatments to delay the onset of resistance and prolong treatment $[5,9]$.

Several studies implicate increased PI3K/Akt/mTOR signaling in trastuzumab resistance. Trastuzumab inhibits PI3K signaling through an increase in PTEN activity, and this PTEN activation is thought to contribute to the drug's antiproliferative effects [10]. These antiproliferative effects, however, are decreased in cells with reduced PTEN expression, suggesting that a reduction in PTEN expression confers trastuzumab resistance [10]. These data are consistent with observations in the clinical setting where PTEN-deficient breast cancers demonstrate poor clinical response to trastuzumab [10]. Mutations leading to PTEN loss or PI3K catalytic subunit activation, both effectively increasing PI3K signaling, are also associated with trastuzumab resistance [11]. Finally, screening of breast cancer patients for genes involved in drug resistance has shown that oncogenic mutations in PI3K catalytic subunit or low PTEN expression are associated with poor prognosis after trastuzumab therapy [12].

The PI3K/Akt/mTOR pathway also plays an important role in modulating responses to estrogen receptor (ER) therapy in a ligand-independent fashion, with sev- 
eral studies indicating that overactivation of Akt, and the subsequent overactivation of downstream mTOR, underlies resistance to endocrine therapies [13-15]. In breast cancer patients, activation of Akt is associated with a worse outcome among patients receiving endocrine therapy, with reduced clinical benefit in patients with positive expression of activated Akt [13]. Other studies have also shown an inverse correlation between Akt activation and partial response (PR) rates. [16]. Expression of phosphorylated S6 kinase, a downstream marker of mTOR activation, significantly predicts overall survival (higher expression equating to poorer survival) in patients with hormone receptor-positive breast cancer receiving adjuvant endocrine therapy [15]. This is mimicked in vitro and in xenograft studies, where breast cancer cells with constitutive Akt activation exhibit reduced estrogen dependency and also demonstrate reduced sensitivity to antiestrogen therapy [14]. In these studies, mTOR inhibition restored antiestrogen sensitivity [15].

\section{Overview of mTOR Inhibitors in Breast Cancer}

\section{Temsirolimus}

\section{Preclinical Studies}

Several in vitro studies report antitumor effects associated with temsirolimus in ER-positive, ER-negative and HER2-positive preclinical breast cancer models $[17,18]$. In MCF-7 breast cancer cells, temsirolimus exhibited synergistic inhibition of cell growth with the ER antagonist, ERA-923, whereas only partial activity was seen with either agent alone. Similar synergy was also seen in a female ovariectomized athymic nu/nu mouse xenograft model, with the combination of drugs inhibiting tumor growth by approximately $85 \%$ compared with control [17].

\section{Clinical History}

Initial dose-escalation studies of temsirolimus assessed doses ranging between $25-100 \mathrm{mg}$ administered orally on days $1-5$ of a 14-day cycle in patients with advanced solid tumors (table 1) [19-22]. The maximum tolerated dose of $75 \mathrm{mg}$ was associated with a time to maximum plasma concentration $\left(\mathrm{T}_{\max }\right)$ of $2.9 \mathrm{~h}$, a maximum plasma concentration $\left(\mathrm{C}_{\max }\right)$ of $23.0 \mathrm{ng} / \mathrm{ml}$ and a terminal half-life of $9.5 \mathrm{~h}$, yielding an area under the plasma concentration-time curve from 0 to $24 \mathrm{~h}\left(\mathrm{AUC}_{0-24 \mathrm{~h}}\right)$ of $147 \mathrm{ng} \cdot \mathrm{h} / \mathrm{ml}$. Overall, oral bioavailability was low at $1.5-2.5 \%$, but with the additional contribution of the ac- tive component, sirolimus, the relative exposure of the oral formulation was $8.8-26.5 \%$ [19].

Overall, 6 of 12 patients receiving temsirolimus $75 \mathrm{mg}$ and 5 of 6 receiving temsirolimus $100 \mathrm{mg}$ required dose reductions [19]. The most frequent adverse events (AEs) leading to dose reduction were mucositis, rash and thrombocytopenia. Ten patients experienced grade 3 AEs. Of 24 enrolled patients, 19 were also evaluable for tumor response. There was no complete response (CR) or PR noted, although 5 patients receiving 75- or 100-mg doses had radiographic minor responses that lasted 8-21 weeks and 2 had stable disease (SD) lasting 35-36 weeks. Overall median time to tumor progression (TTP) was 15.7 weeks [19].

A subsequent phase 2 study indicated that addition of temsirolimus to letrozole may extend progression-free survival (PFS) compared with letrozole alone in patients with locally advanced or metastatic breast cancer (table 1) [20]. Objective responses were reported in 9 patients receiving daily temsirolimus (all PR), 9 patients receiving intermittent temsirolimus (1 CR and 8 PR) and 12 patients receiving letrozole alone (2 CR and $10 \mathrm{PR}$ ); PFS at 1 year was 69, 62 and $48 \%$ in the daily, intermittent and monotherapy arms, respectively [20]. There were no grade $3 / 4$ toxicities among patients receiving letrozole alone; however, in the temsirolimus arms, grade $3 / 4 \mathrm{AEs}$ were reported in $\geq 5 \%$ of patients.

Unfortunately, these promising phase 2 observations were not confirmed in a subsequent large phase 3 randomized study that allocated 1,112 postmenopausal women to receive placebo or temsirolimus administered orally (30 $\mathrm{mg} /$ day on days $1-5$ of a 14 -day cycle) in combination with letrozole ( $2.5 \mathrm{mg} /$ day) (table 1) [21]. At interim analysis, PFS and objective response rates (27\%) were similar in both treatment arms, and grade 3/4 AEs were more common among patients receiving temsirolimus plus letrozole than those receiving letrozole alone (34 vs. 23\%). The study was subsequently terminated based on the recommendation of the Data Safety Monitoring Committee.

\section{Recent Advances: Intravenous Temsirolimus}

Further clinical studies were conducted using an intravenous (IV) formulation of temsirolimus suggesting acceptable tolerability associated with a once-weekly 30 -min IV infusion (table 1) [23, 24]. These initial studies were followed by a phase 2 trial in patients with locally advanced or metastatic breast cancer who had received 1 or 2 previous chemotherapies in the advanced or metastatic setting [22]. Patients were randomly assigned to receive temsirolimus 75 or $250 \mathrm{mg}$ once weekly as a $30-\mathrm{min}$ IV infusion for 6 months or until disease progression. 
Table 1. Clinical trials with temsirolimus in breast cancer [19-22]

\begin{tabular}{|c|c|c|c|c|c|c|}
\hline Study & Study design & Patients, $\mathrm{n}$ & Treatments & $\begin{array}{l}\text { Objective response } \\
\text { rates }\end{array}$ & Disease progression & Safety \\
\hline $\begin{array}{l}\text { Buckner } \\
\text { et al., } \\
2010[19]\end{array}$ & $\begin{array}{l}\text { phase } 1 \text {, } \\
\text { dose-ranging } \\
\text { study }\end{array}$ & $\begin{array}{l}24 \text { patients with } \\
\text { advanced solid } \\
\text { tumors }\end{array}$ & $\begin{array}{l}\text { temsirolimus } 25-100 \mathrm{mg} \\
\text { orally once daily for } 5 \text { days } \\
\text { every } 2 \text { weeks }\end{array}$ & $\begin{array}{l}\mathrm{SD}, \mathrm{n}=2 \\
\text { ( } 5 \text { minor responses) }\end{array}$ & $\begin{array}{l}\text { median TTP = } \\
15.7 \text { weeks }\end{array}$ & $\begin{array}{l}\text { MTD }=75 \mathrm{mg} \\
\text { grade } 3 \text { toxicities of rash and } \\
\text { elevated aminotransferases at } \\
\text { 100-mg dose. Most common } \\
\text { AEs were mucositis, rash/ } \\
\text { maculopapular rash and asthenia }\end{array}$ \\
\hline \multirow[t]{3}{*}{$\begin{array}{l}\text { Carpenter } \\
\text { et al., } \\
2005[20]\end{array}$} & \multirow[t]{3}{*}{$\begin{array}{l}\text { phase } 2 \text {, } \\
\text { randomized }\end{array}$} & \multirow[t]{3}{*}{$\begin{array}{l}\text { locally advanced } \\
\text { BC or } \mathrm{MBC} \\
(\sim 30 \text { patients/arm })\end{array}$} & $\begin{array}{l}\text { letrozole }(2.5 \mathrm{mg} / \text { day })+ \\
\text { temsirolimus } \\
\text { (oral } 10 \mathrm{mg} / \text { day })\end{array}$ & $\mathrm{PR}, \mathrm{n}=9$ & PFS at 1 year: $69 \%$ & $\begin{array}{l}\text { grade } 3 / 4 \text { AEs: } \\
\text { - hyperglycemia, } 9 \%\end{array}$ \\
\hline & & & $\begin{array}{l}\text { letrozole }(2.5 \mathrm{mg} / \text { day })+ \\
\text { temsirolimus (oral } 30 \mathrm{mg} \\
\text { for } 5 \text { days every } 2 \text { weeks) }\end{array}$ & $\mathrm{CR}, \mathrm{n}=1 ; \mathrm{PR}, \mathrm{n}=8$ & PFS at 1 year: $62 \%$ & $\begin{array}{l}\text { grade } 3 / 4 \text { AEs: } \\
\text { - hyperglycemia, } 17 \% \\
\text { - asthenia, } 7 \% \\
\text { - hypertension, } 7 \% \\
\text { - hypokalemia, } 7 \%\end{array}$ \\
\hline & & & letrozole (2.5 mg/day) & $\mathrm{CR}, \mathrm{n}=2 ; \mathrm{PR}, \mathrm{n}=10$ & PFS at 1 year: $48 \%$ & grade 3/4 AEs: none \\
\hline \multirow[t]{2}{*}{$\begin{array}{l}\text { Chow } \\
\text { et al., } \\
2006[21]\end{array}$} & \multirow[t]{2}{*}{$\begin{array}{l}\text { phase } 3, \\
\text { randomized, } \\
\text { placebo- } \\
\text { controlled }\end{array}$} & \multirow[t]{2}{*}{$\begin{array}{l}\text { postmenopausal } \\
\text { women with } \\
\text { locally advanced } \\
\text { BC or MBC, } \\
\mathrm{n}=1,112\end{array}$} & $\begin{array}{l}\text { letrozole }(2.5 \mathrm{mg} / \text { day })+ \\
\text { temsirolimus (oral } 30 \mathrm{mg} / \\
\text { day for } 5 \text { days every } 2 \text { weeks }) \\
(\mathrm{n}=556)\end{array}$ & $\begin{array}{l}\text { CR, } 2 \% ; \mathrm{PR}, 25 \% \\
\mathrm{SD}, 18 \%\end{array}$ & $\begin{array}{l}\text { median PFS: } \\
8.8 \text { months } \\
\text { (95\% CI: } \\
7.4-9.6 \text { months) }\end{array}$ & $\begin{array}{l}\text { grade } 3 / 4 \text { AEs }=34 \% \\
\text { - hyperglycemia, } 4 \% \\
\text { - dyspnea, } 3 \% \\
\text { - neutropenia, 3\% } \\
\text { - asthenia, 3\% }\end{array}$ \\
\hline & & & $\begin{array}{l}\text { letrozole }(2.5 \mathrm{mg} / \text { day })+ \\
\text { placebo }(\mathrm{n}=556)\end{array}$ & $\begin{array}{l}\text { CR, } 2 \% ; \mathrm{PR}, 25 \% \\
\mathrm{SD}, 19 \%\end{array}$ & $\begin{array}{l}\text { median PFS: } \\
8.9 \text { months } \\
\text { (95\% CI: } \\
6.9-9.4 \text { months) }\end{array}$ & $\begin{array}{l}\text { grade } 3 / 4 \text { AEs }=23 \% \\
\text { - dyspnea, } 3 \% \\
\text { - asthenia, } 2 \% \\
\text { - hyperglycemia, } 1 \% \\
\text { - neutropenia, } 1 \%\end{array}$ \\
\hline \multirow[t]{2}{*}{$\begin{array}{l}\text { Chan } \\
\text { et al., } \\
2005 \text { [22] }\end{array}$} & \multirow[t]{2}{*}{$\begin{array}{l}\text { phase 2, } \\
\text { open-label, } \\
\text { 2-dose level, } \\
\text { Simon 2-stage }\end{array}$} & \multirow[t]{2}{*}{$\begin{array}{l}\text { heavily pretreated } \\
\text { locally advanced } \\
\text { BC or MBC, } \\
\mathrm{n}=109\end{array}$} & $\begin{array}{l}\text { temsirolimus } 75 \mathrm{mg} \text { IV via } \\
30 \text {-min infusion once weekly } \\
\text { for } 6 \text { months or until disease } \\
\text { progression }\end{array}$ & & $\begin{array}{l}\text { median TTP }= \\
9.9 \text { weeks }\end{array}$ & $\begin{array}{l}\text { grade } 3 / 4 \text { TEAEs = 53\% } \\
\text { - leukopenia, } 9 \% \\
\text { - GGT increase, } 7 \% \\
\text { - mucositis, } 6 \% \\
\text { - hyperglycemia, } 6 \% \\
\text { - thrombocytopenia, } 6 \% \\
\text { - hypercholesterolemia, } 6 \%\end{array}$ \\
\hline & & & $\begin{array}{l}\text { temsirolimus } 250 \mathrm{mg} \text { IV via } \\
30 \text {-min infusion once } \\
\text { weekly for } 6 \text { months or } \\
\text { until disease progression }\end{array}$ & $\mathrm{PR}, \mathrm{n}=4 ; \mathrm{SD}, \mathrm{n}=3$ & $\begin{array}{l}\text { median TTP }= \\
14.3 \text { weeks }\end{array}$ & $\begin{array}{l}\text { grade } 3 / 4 \text { TEAEs = 57\% } \\
\text { - mucositis, } 14 \% \\
\text { - somnolence, } 10 \% \\
\text { - depression, } 10 \% \\
\text { - hyperglycemia, } 8 \% \\
\text { - asthenia, } 6 \% \\
\text { - anorexia, } 6 \% \\
\text { - vomiting, } 6 \%\end{array}$ \\
\hline
\end{tabular}

AEs = Adverse events; $\mathrm{BC}=$ breast cancer; $\mathrm{CR}=$ complete response; $\mathrm{GGT}=$ gamma glutamyl transferase $; \mathrm{IV}=$ intravenous; $\mathrm{MBC}=\mathrm{metastatic}$ breast cancer; $\mathrm{MTD}=$ maximum tolerated dose; $\mathrm{PFS}=$ progression-free survival; $\mathrm{PR}=$ partial response; $\mathrm{SD}=$ stable disease; $\mathrm{TEAE}=$ treatment emergent $\mathrm{AE}$; TTP = time to tumor progression.

AEs were more common at the 250-mg dose with more dose reductions, treatment delays, higher number of patients with grade $3 / 4$ mucositis ( 7 vs. 3 ), somnolence ( 5 vs. 1) and depression (5 vs. 0 ), and more drug discontinuation. However, antitumor activity was comparable with similar numbers of patients in the 75- and 250-mg dose groups achieving PR ( 6 vs. 4 ) and SD $\geq 24$ weeks ( 2 vs. 3 ). Across both dose groups, median TTP was 12 weeks.

\section{Everolimus}

\section{Preclinical Studies}

Data indicating that overactivation in the PI3K/Akt/ mTOR pathway may underlie resistance to trastuzumab and ER therapy are supported by preclinical studies demonstrating that selective inhibition of mTOR signaling with everolimus can restore sensitivity to these 
agents. Preclinical studies confirmed that everolimus alone exhibits potent antiproliferative and antitumor activity in models of breast cancer in vitro and in vivo [25]. Everolimus also rescued trastuzumab-mediated growth inhibition in cell culture and xenograft models made resistant to trastuzumab through PTEN antisense [26]. Similar observations have also been made regarding resistance to ER therapy. The Akt-mediated resistance to ER therapy seen in breast cancer cells is overcome through co-administration of ER therapies with everolimus [27].

\section{Pharmacokinetics}

Everolimus was rapidly absorbed with a $\mathrm{T}_{\max }$ of $0.5-$ $2.5 \mathrm{~h}$ [28]; however, absorption was delayed by concurrent administration with a high-fat meal. Under these conditions, $\mathrm{T}_{\max }$ was delayed by $1.75 \mathrm{~h}$ and $\mathrm{C}_{\max }$ and AUC were reduced by approximately 50 and $20 \%$, respectively [29]. Exposure was dose proportional over the therapeutic dose range (2.5-25 mg), yielding AUC values of 344-2400 $\mu \mathrm{g} \cdot \mathrm{h} / \mathrm{l}$, with steady-state concentrations achieved after approximately 7 days [28]. Metabolism of everolimus occurred primarily in the gut and liver by cytochrome $\mathrm{P} 450$ $3 \mathrm{~A} 4,3 \mathrm{~A} 5$ and $2 \mathrm{C} 8$, with the majority of drug (approximately $98 \%$ ) excreted in the bile in the form of metabolites [28].

\section{Clinical Studies: Efficacy}

Monotherapy. Everolimus has been evaluated in multiple studies of patients with breast cancer (table 2) [3043]. In a phase 2 study of 49 women with recurrent or metastatic breast cancer, response rates were higher with daily $(10 \mathrm{mg} /$ day) compared with weekly (70 $\mathrm{mg}$ once weekly) oral administration of everolimus. Among the 33 patients receiving daily therapy, there were $1 \mathrm{CR}$ and 3 PRs, with median durations of 13.1 and 3.7 months, respectively [30]. A further 15 patients receiving daily everolimus achieved SD [30].

In a second ongoing monotherapy study, everolimus (10 mg/day) was administered for 8 weeks to patients with invasive breast cancer and bone metastases [31]. Of 52 patients reported so far, 2 patients had a CR or PR and continued on treatment and 32 patients had progressive disease and were discontinued. The remaining 18 patients with SD were randomly allocated to continue with everolimus or receive placebo and the study is ongoing [31].

Everolimus Studies in Hormone Receptor-Positive Patients. Recent data from the Breast Cancer Trial of Oral Everolimus (BOLERO)-2 study indicates that addition of everolimus to exemestane significantly improved PFS in patients with hormone receptor-positive advanced breast cancer (table 2) [32]. BOLERO-2 was a phase 3, placebocontrolled, randomized study comparing everolimus plus exemestane versus placebo plus exemestane in 724 patients with hormone receptor-positive advanced breast cancer refractory to anastrozole or letrozole. Median PFS was 6.9 months in patients receiving everolimus versus 2.8 months in the placebo arm (hazard ratio $[\mathrm{HR}]=0.43$, 95\% CI: 0.35-0.54). Objective response rates were 9.5\% with everolimus and $0.4 \%$ with placebo $(\mathrm{p}<0.001)$ [32]. A longer-term prespecified 12-month follow-up analysis of BOLERO-2 confirmed these findings. Median PFS was 7.4 months versus 3.2 months, and objective response rates were 12.0 and $1.3 \%$ for combination and exemestane-only therapy, respectively ( $\mathrm{HR}=0.44,95 \% \mathrm{CI}$ : 0.36 $0.53, p<0.001$ [local assessment]) [33]. The median dose intensity (cumulative dose/duration of exposure) of everolimus was $8.6 \mathrm{mg}$ (range: $0.3-10 \mathrm{mg}$ ) and PFS was similar regardless of whether or not a patient's dose intensity during the study was reduced, possibly due to adverse events $(<7.5 \mathrm{mg} /$ day, $\mathrm{HR}=0.40,95 \% \mathrm{CI}: 0.31-0.52$; $\geq 7.5 \mathrm{mg} /$ day, $\mathrm{HR}=0.45,95 \%$ CI: 0.37-0.56) [44]. Recently, an 18-month follow-up analysis determined that the median PFS was 7.8 months versus 3.2 months ( $\mathrm{HR}=$ $0.45,95 \%$ CI: $0.38-0.54, \mathrm{p}<0.001$ [local assessment]) and objective response rates were 12.6 and $1.7 \%$ for combination and exemestane-only therapy, respectively $(\mathrm{p}<$ 0.001) [34].

Two randomized, phase 2 studies also indicate that the addition of everolimus to endocrine therapy is associated with improved response rates in patients with breast cancer (table 2). TAMRAD evaluated tamoxifen alone (20 mg/day) or in combination with everolimus (10 mg/ day) in patients with hormone receptor-positive and HER2-negative metastatic breast cancer previously treated with aromatase inhibitor therapy [35]. After a median follow-up of approximately 24 months, the clinical benefit rate (CR plus PR plus SD at 6 months) was $42 \%$ (95\% CI: $29-56)$ versus $61 \%$ (95\% CI: 47-74) in monotherapy versus combination treatment arms (exploratory $\mathrm{p}=$ 0.045). Consistent with tumor responses, TTP was significantly longer in the combination arm (8.6 months vs. 4.5 months; $\mathrm{HR}=0.54,95 \% \mathrm{CI}: 0.36-0.81$, exploratory $\mathrm{p}$ $=0.002)$ [35].

In a second phase 2 study, 270 patients with newly diagnosed ER-positive, localized breast cancer were treated with neoadjuvant letrozole $(2.5 \mathrm{mg} /$ day $)$ alone or in combination with everolimus (10 mg/day) [36]. Response rates (by clinical palpation) were $68.1 \%$ (95\% CI: 60.3- 
Table 2. Clinical trials with everolimus in breast cancer [30-43]

\begin{tabular}{|c|c|c|c|c|c|c|}
\hline Study & Study design & Patients, n & Treatments & $\begin{array}{l}\text { Objective } \\
\text { response rates }\end{array}$ & $\begin{array}{l}\text { Disease } \\
\text { progression }\end{array}$ & Safety \\
\hline \multicolumn{7}{|c|}{ Everolimus monotherapy studies } \\
\hline \multirow[t]{2}{*}{$\begin{array}{l}\text { Ellard } \\
\text { et al., } \\
2009[30]\end{array}$} & \multirow[t]{2}{*}{$\begin{array}{l}\text { randomized } \\
\text { phase } 2\end{array}$} & \multirow[t]{2}{*}{$\begin{array}{l}\text { minimally } \\
\text { pretreated/ } \\
\text { recurrent } \mathrm{MBC}\end{array}$} & $\begin{array}{l}\text { everolimus } \\
10 \mathrm{mg} / \text { day orally }\end{array}$ & $\begin{array}{l}C R, \mathrm{n}=1 ; \mathrm{PR}, \mathrm{n}=3 \\
\mathrm{SD}, \mathrm{n}=15\end{array}$ & not evaluated & $\begin{array}{l}\text { D/C because of toxicity: } 27 \% \\
\text { - pneumonitis, } n=4 \\
\text { - transaminase elevation, } n=2\end{array}$ \\
\hline & & & $\begin{array}{l}\text { everolimus } \\
70 \mathrm{mg} / \text { week orally }\end{array}$ & $\begin{array}{l}\text { accrual suspended } \\
\text { because of disease } \\
\text { progression rates }\end{array}$ & not evaluated & $\begin{array}{l}\text { D/C because of toxicity: } 13 \% \\
\text { - pneumonitis, } n=1 \\
\text { - fatigue, } n=1\end{array}$ \\
\hline $\begin{array}{l}\text { Maas } \\
\text { et al., } \\
2009[31]\end{array}$ & prospective & $\begin{array}{l}\text { invasive BC } \\
\text { and bone } \\
\text { metastases } \\
(\mathrm{n}=52)\end{array}$ & $\begin{array}{l}\text { everolimus } \\
(10 \mathrm{mg} / \text { day }) \text { for } \\
8 \text { weeks }+ \\
\text { zoledronic acid }\end{array}$ & $\begin{array}{l}\mathrm{CR}, \mathrm{n}=1 ; \mathrm{PR}, \mathrm{n}=1 ; \\
\mathrm{PD}, \mathrm{n}=32 \\
\text { no change at } 8 \text { weeks, } \\
\mathrm{n}=18^{*}\end{array}$ & not evaluated & $\begin{array}{l}\text { SAEs, } \mathrm{n}=14 \\
\text { - diarrhea/vomiting, } \mathrm{n}=4 \\
\text { - infection, } \mathrm{n}=2 \\
\text { - anemia/pancytopenia, } \mathrm{n}=2\end{array}$ \\
\hline \multicolumn{7}{|c|}{ Everolimus in patients with hormone receptor-positive disease } \\
\hline $\begin{array}{l}\text { Baselga } \\
\text { et al., } \\
2012^{\dagger}[32]\end{array}$ & \multirow[t]{2}{*}{$\begin{array}{l}\text { placebo- } \\
\text { controlled, } \\
\text { randomized } \\
\text { phase } 3\end{array}$} & \multirow[t]{2}{*}{$\begin{array}{l}\text { postmenopausal } \\
\text { ER-positive } \\
\text { advanced BC } \\
(\mathrm{n}=724)\end{array}$} & $\begin{array}{l}\text { exemestane } \\
(25 \mathrm{mg} / \text { day })+ \\
\text { everolimus } \\
\text { (10 } \mathrm{mg} / \text { day })\end{array}$ & $\begin{array}{l}\text { CR, } 0.4 \% ; \mathrm{PR}, 9.1 \% \\
\text { SD, } 70.1 \% \\
\text { ORR, } 9.5 \%\end{array}$ & $\mathrm{PFS}=6.9$ months & $\begin{array}{l}\text { grade } 3 / 4 \text { AEs } \\
\text { - stomatitis, } 8 \% \\
\text { - anemia, } 6 \% \\
\text { - hyperglycemia, } 4 \% \\
\text { - dyspnea, } 4 \% \\
\text { - fatigue, } 4 \% \\
\text { D/C because of AE, } 19 \%\end{array}$ \\
\hline BOLERO-2 & & & $\begin{array}{l}\text { exemestane } \\
(25 \mathrm{mg} / \text { day })+ \\
\text { placebo }\end{array}$ & $\begin{array}{l}\text { CR, } 0 \% ; \text { PR, } 0.4 \% \\
\text { SD, } 58.6 \% \\
\text { ORR, } 0.4 \% \\
\text { (p<0.001 vs. } \\
\text { exemestane alone) }\end{array}$ & $\begin{array}{l}\text { PFS }=2.8 \text { months } \\
(\mathrm{HR}=0.43 ; 95 \% \mathrm{CI}: \\
0.35-0.54)\end{array}$ & $\begin{array}{l}\text { grade } 3 / 4 \text { AEs } \\
\text { - stomatitis, } 1 \% \\
\text { - anemia, }<1 \% \\
\text { - hyperglycemia, }<1 \% \\
\text { - dyspnea, } 1 \% \\
\text { - fatigue, } 1 \% \\
\text { D/C because of AE, } 4 \%\end{array}$ \\
\hline $\begin{array}{l}\text { Hortobagyi } \\
\text { et al., } \\
2011^{\dagger}\end{array}$ & $\begin{array}{l}\text { placebo- } \\
\text { controlled, } \\
\text { randomized } \\
\text { phase } 3\end{array}$ & $\begin{array}{l}\text { postmenopausal } \\
\text { ER-positive } \\
\text { advanced BC } \\
(\mathrm{n}=724)\end{array}$ & $\begin{array}{l}\text { exemestane } \\
(25 \mathrm{mg} / \text { day })+ \\
\text { everolimus } \\
(10 \mathrm{mg} / \text { day })\end{array}$ & ORR, $12.0 \%$ & $\mathrm{PFS}=7.4$ months & $\begin{array}{l}\text { grade } 3 / 4 \text { AEs } \\
\text { - stomatitis, } 8 \% \\
\text { - anemia, } 7 \% \\
\text { - hyperglycemia, } 5 \% \\
\text { - dyspnea, } 4 \% \\
\text { - fatigue, } 4 \% \\
\text { D/C because of AE, } 8 \%\end{array}$ \\
\hline $\begin{array}{l}\text { BOLERO-2 } \\
\text { (12-month } \\
\text { data update) }\end{array}$ & & & $\begin{array}{l}\text { exemestane } \\
(25 \mathrm{mg} / \text { day })+ \\
\text { placebo }\end{array}$ & $\begin{array}{l}\text { ORR, } 1.3 \% \\
(\mathrm{p}<0.0001 \text { vs. } \\
\text { exemestane alone })\end{array}$ & $\begin{array}{l}\text { PFS }=3.2 \text { months } \\
(\mathrm{HR}=0.44 ; 95 \% \mathrm{CI}: \\
0.36-0.53)\end{array}$ & $\begin{array}{l}\text { grade } 3 / 4 \text { AEs } \\
\text { - stomatitis, } 1 \% \\
\text { - anemia, } 1 \% \\
\text { - hyperglycemia, <1\% } \\
\text { - dyspnea, } 1 \% \\
\text { - fatigue, } 1 \% \\
\text { D/C because of AE, 3\% }\end{array}$ \\
\hline $\begin{array}{l}\text { Piccart } \\
\text { et al., } \\
2012^{\dagger}[34]\end{array}$ & \multirow[t]{2}{*}{$\begin{array}{l}\text { placebo- } \\
\text { controlled, } \\
\text { randomized } \\
\text { phase } 3\end{array}$} & \multirow[t]{2}{*}{$\begin{array}{l}\text { postmenopausal } \\
\text { ER-positive } \\
\text { advanced BC } \\
(\mathrm{n}=724)\end{array}$} & $\begin{array}{l}\text { exemestane } \\
(25 \mathrm{mg} / \text { day })+ \\
\text { everolimus } \\
(10 \mathrm{mg} / \text { day })\end{array}$ & ORR, $12.6 \%$ & $\mathrm{PFS}=7.8$ months & $\begin{array}{l}\text { grade } 3 / 4 \text { AEs } \\
\text { - stomatitis, } 8 \% \\
\text { - hyperglycemia, } 5 \% \\
\text { - fatigue, } 4 \% \\
\text { D/C because of AE, } 9 \%\end{array}$ \\
\hline $\begin{array}{l}\text { BOLERO-2 } \\
\text { (18-month } \\
\text { data update) }\end{array}$ & & & $\begin{array}{l}\text { exemestane } \\
(25 \mathrm{mg} / \text { day })+ \\
\text { placebo }\end{array}$ & $\begin{array}{l}\text { ORR, } 1.7 \% \\
(p<0.0001 \text { vs. } \\
\text { exemestane alone })\end{array}$ & $\begin{array}{l}\text { PFS }=3.2 \text { months } \\
(\mathrm{HR}=0.45 ; 95 \% \mathrm{CI}: \\
0.38-0.54)\end{array}$ & $\begin{array}{l}\text { grade } 3 / 4 \text { AEs } \\
\text { - stomatitis, } 1 \% \\
\text { - hyperglycemia, }<1 \% \\
\text { - fatigue, } 1 \% \\
\text { D/C because of AE, } 3 \%\end{array}$ \\
\hline $\begin{array}{l}\text { Bachelot, } \\
\text { et al., } \\
2012[35]\end{array}$ & $\begin{array}{l}\text { randomized } \\
\text { phase 2, } \\
\text { Simon 2-stage }\end{array}$ & $\begin{array}{l}\text { hormone- } \\
\text { receptor-positive, } \\
\text { HER2-negative } \\
\text { MBC }\end{array}$ & $\begin{array}{l}\text { tamoxifen } \\
(20 \mathrm{mg} / \text { day })+ \\
\text { everolimus } \\
(10 \mathrm{mg} / \text { day })(\mathrm{n}=54)\end{array}$ & CBR, $61 \%$ & $\mathrm{TTP}=8.6$ months & $\begin{array}{l}\text { grade } 3 / 4 \text { AEs } \\
\text { - stomatitis, } 11 \% \\
\text { - anorexia, } 7 \% \\
\text { - pain, } 9 \% \\
\text { - rash, } 4 \%\end{array}$ \\
\hline TAMRAD & & & $\begin{array}{l}\text { tamoxifen } \\
(20 \mathrm{mg} / \text { day }) \\
(\mathrm{n}=57)\end{array}$ & $\begin{array}{l}\text { CBR, } 42 \% \\
\text { (exploratory } \\
p=0.045 \\
\text { vs. tamoxifen alone) }\end{array}$ & $\begin{array}{l}\text { TTP }=4.5 \text { months } \\
\text { (exploratory } \\
\mathrm{p}=0.002 \text { ) }\end{array}$ & $\begin{array}{l}\text { grade } 3 / 4 \text { AEs } \\
\text { - pain, } 18 \% \\
\text { - fatigue, } 11 \% \\
\text { - infection, } 5 \%\end{array}$ \\
\hline
\end{tabular}


Table 2 (continued)

\begin{tabular}{|c|c|c|c|c|c|c|}
\hline Study & Study design & Patients, n & Treatments & $\begin{array}{l}\text { Objective } \\
\text { response rates }\end{array}$ & $\begin{array}{l}\text { Disease } \\
\text { progression }\end{array}$ & Safety \\
\hline \multirow[t]{2}{*}{$\begin{array}{l}\text { Baselga, } \\
\text { et al., } \\
2009[36]\end{array}$} & \multirow[t]{2}{*}{$\begin{array}{l}\text { phase } 2 \\
\text { randomized }\end{array}$} & \multirow[t]{2}{*}{$\begin{array}{l}\text { postmenopausal } \\
\text { women with } \\
\text { operable } \\
\text { ER-positive BC } \\
(\mathrm{n}=270)\end{array}$} & $\begin{array}{l}\text { everolimus } \\
(10 \mathrm{mg} / \text { day })+ \\
\text { neoadjuvant letrozole } \\
(2.5 \mathrm{mg} / \text { day }) \\
(\mathrm{n}=138)\end{array}$ & $\begin{array}{l}\text { CR, } 18(13.0 \%) \\
\text { PR, } 76(55.1 \%) \\
\mathrm{ORR}^{\ddagger}=68.1 \% \\
(95 \% \mathrm{CI}: 60.3-75.9)\end{array}$ & not evaluated & $\begin{array}{l}\text { grade } 3 / 4 \text { AEs, } \mathrm{n}=31 \\
\text { - stomatitis, } \mathrm{n}=3 \\
\text { - hyperglycemia, } \mathrm{n}=7 \\
\text { - pneumonitis, } \mathrm{n}=3 \\
\text { dose reduction/interruption, } 52.9 \%\end{array}$ \\
\hline & & & $\begin{array}{l}\text { neoadjuvant letrozole } \\
(2.5 \mathrm{mg} / \text { day })+\text { placebo } \\
(\mathrm{n}=132)\end{array}$ & $\begin{array}{l}\text { CR, } 12(9.1 \%) \\
\text { PR, } 66(50 \%) \\
\mathrm{ORR}^{\ddagger}=59.1 \% \\
(95 \% \text { CI: } 50.7-67.5)\end{array}$ & not evaluated & $\begin{array}{l}\text { grade } 3 / 4 \text { AEs, } n=5 \\
\text { - asthenia, } n=1 \\
\text { - arthralgia, } n=1 \\
\text { - cellulitis, } n=1 \\
\text { dose reduction/interruption, } 7.6 \%\end{array}$ \\
\hline $\begin{array}{l}\text { Badin, } \\
\text { et al., } \\
2010 \text { [37] }\end{array}$ & $\begin{array}{l}\text { phase } 2 \\
\text { open-label }\end{array}$ & $\begin{array}{l}\text { ER-positive } \\
\text { MBC with } \\
\text { aromatase } \\
\text { inhibitor } \\
\text { therapy failure } \\
\text { within } 6 \text { months } \\
(\mathrm{n}=11)\end{array}$ & $\begin{array}{l}\text { everolimus } \\
(10 \mathrm{mg} / \text { day })+ \\
\text { fulvestrant } \\
(500 \mathrm{mg} \text { day } 1, \\
250 \mathrm{mg} \text { day } 14, \\
250 \mathrm{mg} \text { day } 28 \text { and } \\
\text { monthly thereafter) }\end{array}$ & $\mathrm{CBR}=55 \%$ & $\mathrm{TTP}=8.6$ months & $\begin{array}{l}\text { mucositis, } 63.6 \% \\
\text { rash, } 45.5 \% \\
\text { infection, } 36.4 \% \\
\text { fatigue, } 27.3 \% \\
\text { most of the toxicities were grade } 1 \\
\text { no grade } 4 \text { toxicities were observed }\end{array}$ \\
\hline \multicolumn{7}{|c|}{ Everolimus in patients overexpressing HER2 } \\
\hline \multirow[t]{2}{*}{$\begin{array}{l}\text { Jerusalem } \\
\text { et al., } \\
2011[38]\end{array}$} & \multirow[t]{2}{*}{$\begin{array}{l}\text { phase } 1 \mathrm{~b}, \\
\text { dose-escalation } \\
\text { study }\end{array}$} & \multirow[t]{2}{*}{$\begin{array}{l}\text { HER2- } \\
\text { overexpressing } \\
\text { MBC }\end{array}$} & $\begin{array}{l}\text { everolimus } 5 \mathrm{mg} / \mathrm{day} \\
+ \text { trastuzumab } \pm \\
\text { vinorelbine }(\mathrm{n}=30)\end{array}$ & $\begin{array}{l}\mathrm{CR}, \mathrm{n}=1 \\
\mathrm{PR}, \mathrm{n}=5 \\
\mathrm{SD}, \mathrm{n}=18\end{array}$ & $\begin{array}{l}\text { median PFS: } \\
\text { 30.7 weeks } \\
\text { (95\% CI: } 28-44.9)\end{array}$ & $\begin{array}{l}\text { grade } 3 / 4 \text { AEs } \\
\text { - neutropenia, } n=25 \\
\text { - leukopenia, } \mathrm{n}=14 \\
\text { - stomatitis, } \mathrm{n}=5\end{array}$ \\
\hline & & & $\begin{array}{l}\text { everolimus } \\
20-30 \mathrm{mg} / \mathrm{week}+ \\
\text { trastuzumab } \pm \\
\text { vinorelbine }(\mathrm{n}=20)\end{array}$ & $\begin{array}{l}\mathrm{CR}, \mathrm{n}=0 \\
\mathrm{PR}, \mathrm{n}=3 \\
\mathrm{SD}, \mathrm{n}=12\end{array}$ & $\begin{array}{l}\text { median PFS: } \\
27.1 \text { weeks } \\
\text { (95\% CI: } 25.6-N / A)\end{array}$ & $\begin{array}{l}\text { grade } 3 / 4 \text { AEs } \\
\text { - neutropenia, } n=18 \\
\text { - leukopenia, } n=8 \\
\text { - lymphopenia, } n=3\end{array}$ \\
\hline \multirow[t]{2}{*}{$\begin{array}{l}\text { Andre } \\
\text { et al., } \\
2010 \text { [39] }\end{array}$} & \multirow[t]{2}{*}{$\begin{array}{l}\text { phase } 1 b \text {, } \\
\text { dose-escalation } \\
\text { study }\end{array}$} & \multirow[t]{2}{*}{$\begin{array}{l}\text { MBC pretreated } \\
\text { with } \\
\text { trastuzumab }\end{array}$} & $\begin{array}{l}\text { everolimus } \\
(5-10 \mathrm{mg} / \text { day })+ \\
\text { paclitaxel } / \\
\text { trastuzumab }(\mathrm{n}=23)\end{array}$ & $\begin{array}{l}\mathrm{CR}, \mathrm{n}=2 \\
\mathrm{PR}, \mathrm{n}=7 \\
\mathrm{SD}, \mathrm{n}=8\end{array}$ & $\begin{array}{l}\text { median PFS: } \\
33 \text { weeks } \\
\text { (95\% CI: } 23.7-N / A)\end{array}$ & $\begin{array}{l}\text { grade } 3 / 4 \text { AEs } \\
\text { - neutropenia, } \mathrm{n}=13 \\
\text { - leukopenia, } \mathrm{n}=8 \\
\text { - lymphopenia, } \mathrm{n}=7 \\
\text { - stomatitis, } \mathrm{n}=4\end{array}$ \\
\hline & & & $\begin{array}{l}\text { everolimus } \\
(30 \mathrm{mg} / \text { week })+ \\
\text { paclitaxel } / \\
\text { trastuzumab }(\mathrm{n}=10)\end{array}$ & $\begin{array}{l}\mathrm{CR}, \mathrm{n}=0 \\
\mathrm{PR}, \mathrm{n}=3 \\
\mathrm{SD}, \mathrm{n}=5\end{array}$ & $\begin{array}{l}\text { median PFS: } \\
40.7 \text { weeks } \\
\text { (95\% CI: } 30-N / A)\end{array}$ & $\begin{array}{l}\text { grade } 3 / 4 \text { AEs } \\
\text { - neutropenia, } \mathrm{n}=4 \\
\text { - leukopenia, } \mathrm{n}=2 \\
\text { - lymphopenia, } \mathrm{n}=4 \\
\text { - stomatitis, } \mathrm{n}=3\end{array}$ \\
\hline $\begin{array}{l}\text { Dalenc } \\
\text { et al., } \\
2010[40]\end{array}$ & $\begin{array}{l}\text { phase } 2 \\
\text { Simon 2-stage }\end{array}$ & $\begin{array}{l}\text { HER2- } \\
\text { overexpressing } \\
\text { MBC resistant } \\
\text { to taxanes and } \\
\text { trastuzumab }\end{array}$ & $\begin{array}{l}\text { everolimus } \\
(10 \mathrm{mg} / \mathrm{day})+ \\
\text { paclitaxel/ } \\
\text { trastuzumab }(\mathrm{n}=55)\end{array}$ & $\begin{array}{l}\mathrm{CR}, \mathrm{n}=0 \\
\mathrm{PR}, \mathrm{n}=9 \\
\mathrm{SD}, \mathrm{n}=30\end{array}$ & $\begin{array}{l}\text { median PFS: } \\
26 \text { weeks } \\
(95 \% \text { CI: } 23.1-40.4)\end{array}$ & $\begin{array}{l}\text { grade } 3 / 4 \text { AEs } \\
\text { - neutropenia, } \mathrm{n}=15 \\
\text { - stomatitis, } \mathrm{n}=11 \\
\text { - lymphopenia, } \mathrm{n}=8\end{array}$ \\
\hline $\begin{array}{l}\text { Morrow } \\
\text { et al., } \\
2011[41]\end{array}$ & phase $1 / 2$ & $\begin{array}{l}\text { trastuzumab- } \\
\text { resistant MBC } \\
\text { overexpressing } \\
\text { HER2 }\end{array}$ & $\begin{array}{l}\text { trastuzumab } \\
(8 \mathrm{mg} / \mathrm{kg} \text {, then } \\
6 \mathrm{mg} / \mathrm{kg} \text { every } \\
3 \text { weeks })+ \text { everolimus } \\
(10 \mathrm{mg} / \text { day })(\mathrm{n}=47)\end{array}$ & $\begin{array}{l}\mathrm{PR}, \mathrm{n}=7 \\
\mathrm{SD} \geq 24 \text { weeks, } \\
\mathrm{n}=9\end{array}$ & $\begin{array}{l}\text { median PFS: } \\
4.1 \text { months }\end{array}$ & $\begin{array}{l}\text { grade } 3 / 4 \text { AEs } \\
\text { - lymphopenia, } 13 \% \\
\text { - hyperglycemia, } 13 \% \\
\text { - mucositis, } 9 \%\end{array}$ \\
\hline \multicolumn{7}{|c|}{ Everolimus in patients with HER2-negative disease } \\
\hline $\begin{array}{l}\text { Mayer } \\
\text { et al., } \\
2009 \text { [42] }\end{array}$ & $\begin{array}{l}\text { open-label } \\
\text { phase 1b dose } \\
\text { escalation } \\
\text { study }\end{array}$ & $\begin{array}{l}\text { HER2-negative } \\
\text { MBC } \\
\text { (70\% TNBC) }\end{array}$ & $\begin{array}{l}\text { everolimus } \\
(20-30 \mathrm{mg} / \text { week })+ \\
\text { cisplatin }\left(25 \mathrm{mg} / \mathrm{m}^{2}\right) / \\
\text { paclitaxel }\left(80 \mathrm{mg} / \mathrm{m}^{2}\right) \\
\text { once weekly for } \\
3 \text { weeks in a } 4 \text {-week } \\
\text { cycle }(\mathrm{n}=16)\end{array}$ & $\begin{array}{l}13 \text { patients } \\
\text { evaluable for } \\
\text { response } \\
\quad \text { CR } \mathrm{n}=1 \\
\quad \mathrm{PR}, \mathrm{n}=2 \\
\quad \mathrm{SD}, \mathrm{n}=7 \\
\text { all CR/PRs } \\
\text { receiving } \\
\text { everolimus } \\
30 \text { mg/week) }\end{array}$ & $\begin{array}{l}\text { median TTP: } \\
5 \text { months }\end{array}$ & $\begin{array}{l}\text { grade } 3 / 4 \text { AEs } \\
\text { - neutropenia, } 8.5 \%\end{array}$ \\
\hline
\end{tabular}


Table 2 (continued)

\begin{tabular}{|c|c|c|c|c|c|c|}
\hline Study & Study design & Patients, $\mathrm{n}$ & Treatments & $\begin{array}{l}\text { Objective } \\
\text { response rates }\end{array}$ & $\begin{array}{l}\text { Disease } \\
\text { progression }\end{array}$ & Safety \\
\hline \multirow[t]{2}{*}{$\begin{array}{l}\text { Gonzalez- } \\
\text { Angulo } \\
\text { et al., } \\
2011[43]\end{array}$} & \multirow[t]{2}{*}{$\begin{array}{l}\text { single- } \\
\text { institution } \\
\text { phase } 2\end{array}$} & \multirow[t]{2}{*}{$\begin{array}{l}\text { primary TNBC } \\
\text { in neoadjuvant } \\
\text { setting }\end{array}$} & $\begin{array}{l}\text { paclitaxel for } 12 \text { weeks } \\
\text { followed by } \\
5 \text {-fluorouracil, } \\
\text { epirubicin and } \\
\text { cyclophosphamide } \\
\text { every } 3 \text { weeks for } \\
4 \text { cycles }\end{array}$ & $\begin{array}{l}\text { at week } 12 \\
\qquad \begin{array}{l}\mathrm{CR}, \mathrm{n}=3 \\
\mathrm{PR}, \mathrm{n}=5 \\
\mathrm{SD}, \mathrm{n}=16\end{array} \\
\text { at week } 24 \\
\begin{array}{l}\mathrm{CR}, \mathrm{n}=4 \\
\mathrm{PR}, \mathrm{n}=16 \\
\mathrm{SD}, \mathrm{n}=7\end{array}\end{array}$ & not evaluated & $\begin{array}{l}\text { grade } 3 / 4 \text { AEs } \\
\text { - neutropenia, } 41 \% \\
\text { - GI disturbances, 19\% } \\
\text { - increased leukocytes, 11\% } \\
\text { - fatigue, } 7 \% \\
\text { - thrombocytopenia, } 7 \% \\
\text { - pain, } 7 \%\end{array}$ \\
\hline & & & $\begin{array}{l}\text { paclitaxel + everolimus } \\
(30 \mathrm{mg} / \text { week) for } \\
12 \text { weeks followed by } \\
5 \text {-fluorouracil, } \\
\text { epirubicin and } \\
\text { cyclophosphamide } \\
\text { every } 3 \text { weeks for } \\
4 \text { cycles }\end{array}$ & $\begin{array}{l}\text { at week } 12 \\
\qquad \mathrm{CR}, \mathrm{n}=0 \\
\mathrm{PR}, \mathrm{n}=11 \\
\mathrm{SD}, \mathrm{n}=11 \\
\text { at week } 24 \\
\quad \mathrm{CR}, \mathrm{n}=2 \\
\mathrm{PR}, \mathrm{n}=11 \\
\mathrm{SD}, \mathrm{n}=7\end{array}$ & & $\begin{array}{l}\text { grade } 3 / 4 \text { AEs } \\
\text { - neutropenia, 52\% } \\
\text { - GI disturbances, } 26 \% \\
\text { - increased leukocytes, } 17 \% \\
\text { - fatigue, } 17 \% \\
\text { - anemia, } 17 \% \\
\text { - pain, } 13 \% \\
\text { - infection, } 13 \%\end{array}$ \\
\hline \multicolumn{4}{|c|}{$\begin{array}{l}\text { AEs }=\text { Adverse events; } \mathrm{BC}=\text { breast cancer; } \mathrm{CBR}=\text { clinical benefit rate }(\mathrm{CR} \\
+\mathrm{PR}+\mathrm{SD} \text { at } 6 \text { months); } \mathrm{CI}=\text { confidence interval; } \mathrm{CR}=\text { complete response; } \\
\mathrm{D} / \mathrm{C}=\text { discontinuation; } \mathrm{ER}=\text { estrogen receptor; } \mathrm{GI}=\text { gastrointestinal; } \mathrm{HR}= \\
\text { hazard ratio; } \mathrm{HER}=\text { human epidermal growth factor receptor; } \mathrm{MBC}=\text { meta- } \\
\text { static breast cancer; } \mathrm{N} / \mathrm{A}=\text { not available; } \mathrm{ORR}=\text { objective response rate; } \mathrm{PR}\end{array}$} & \multicolumn{3}{|c|}{$\begin{array}{l}=\text { partial response; } \mathrm{SAEs}=\text { serious adverse events; } \mathrm{SD}=\text { stable disease; TNBC } \\
=\text { triple-negative breast cancer; TTP }=\text { time to progression. } \\
\quad{ }^{*} \text { Patients with no change at week } 8 \text { were randomized to continue with } \\
\text { everolimus } 10 \mathrm{mg} / \text { day or receive placebo for further treatment; data not avail- } \\
\text { able. }{ }^{\dagger} \text { Data based on local review. }{ }^{\ddagger} \text { By palpation. }\end{array}$} \\
\hline
\end{tabular}

75.9) in the combination and 59.1\% (95\% CI: 50.7-67.5) in the monotherapy arm (1-sided $\mathrm{p}=0.06)$, meeting the prespecified primary end point according to the 1 -sided $\chi^{2}$ test significance threshold of $\mathrm{p}<0.1$ [36].

Additionally, an open-label phase 2 trial of everolimus $(10 \mathrm{mg} /$ day $)$ in combination with intramuscular fulvestrant is currently underway in patients with ER-positive disease who failed an aromatase inhibitor within 6 months of study entry. The median TTP was 8.6 months and the clinical benefit rate was $55 \%$ in the initial 11 patients enrolled [37].

Everolimus Studies in HER2-Positive Patients. Phase 1 dose escalation studies support the use of everolimus at doses of 5-10 mg/day in combination regimens for metastatic breast cancer (table 2) [38-41]. In combination with trastuzumab and vinorelbine, the most common doselimiting toxicity was neutropenia [38]. Doses of $5 \mathrm{mg} / \mathrm{day}$ and $20-30 \mathrm{mg} /$ week were subsequently evaluated for safety and efficacy in combination with trastuzumab alone ( $6 \mathrm{mg} / \mathrm{kg}$ every 3 weeks) or trastuzumab ( $2 \mathrm{mg} / \mathrm{kg} /$ week) plus vinorelbine. Among 47 evaluable patients, overall response rates (ORRs) were 19\% and disease control rate was $83 \%$, with no marked differences between daily and weekly schedules. Median PFS was 30.7 weeks in the daily everolimus arm and 27.1 weeks in the weekly everolimus arm [38]. A similar phase 1 study identified everolimus $10 \mathrm{mg} / \mathrm{day}$ as the appropriate dose for further investigation when used in combination with paclitaxel and trastuzumab in patients with metastatic breast cancer pretreated with trastuzumab [39]. Median PFS was 34 weeks (95\% CI: 29.1-40.7) and ORR was 44\%. [39]. A follow-up phase 2 study using a similar treatment regimen in 55 patients with metastatic breast cancer resistant to taxanes and trastuzumab yielded an ORR of 19\% (all PR) and an SD rate of $62 \%$. The median PFS was 26 weeks (95\% CI: 23.1-40.4) [40]. Everolimus plus trastuzumab (without chemotherapy) has also demonstrated activity in patients with trastuzumab resistant metastatic breast cancer, with a clinical benefit rate of $34 \%$ and median PFS of 4.1 months [41].

Everolimus Studies in HER2-Negative Patients. Everolimus-containing regimens have been assessed in a small number of patients with HER2-negative metastatic breast cancer (table 2). In a small phase $1 / 2$ study that included 16 patients with HER2-negative metastatic breast cancer receiving everolimus in combination with cisplatin and paclitaxel ( $70 \%$ had triple-negative disease), 1 patient achieved a CR, 2 had a PR and 7 had SD [42]. Median TTP was 5 months [42]. A single-institution study has also evaluated the use of everolimus in patients with earlystage or locally advanced triple-negative disease [43]. In this study, patients were randomized to receive neoadju- 
vant paclitaxel alone $(\mathrm{n}=27)$ or in combination with everolimus ( $30 \mathrm{mg} /$ week; $\mathrm{n}=23$ ) for 12 weeks, followed by 5 -fluorouracil, epirubicin and cyclophosphamide every 3 weeks for 4 cycles. At the end of the initial 12-week dosing period, there was no significant difference in response rates between treatment arms, with 8 of $27 \mathrm{pa}$ tients receiving paclitaxel achieving CR $(n=3)$ or PR (n $=5$ ) and 11 patients receiving paclitaxel plus everolimus achieving PR [43]. There was also no difference in response rates between treatment arms at week 24 , and pathological CRs were also similar [43].

\section{Clinical Studies: Safety}

Everolimus has a predictable safety profile (table 2). In monotherapy studies, fatigue, pneumonitis, infection, neutropenia, diarrhea and vomiting were the most frequently reported grade $3 / 4$ AEs [30, 31]. In 1 study, pneumonitis was reported more frequently with daily $(10 \mathrm{mg} /$ day) compared with weekly (70 mg/week) administration [30]. With daily administration, $46 \%$ of patients developed any-grade pneumonitis, with a median time to onset of 51 days. The pneumonitis was symptomatic (grade $\geq 2$ ) in most patients and reversible in all cases with appropriate measures (drug hold, steroid treatment for grade $\geq 2$, rechallenge at reduced dose in patients who recovered to grade $\leq 1)$. Median duration of symptomatic pneumonitis was 1.9 months [30].

In combination with endocrine therapy, stomatitis, rash, hyperglycemia and pneumonitis were distinguishing AEs in everolimus treatment arms. In BOLERO-2, $23 \%$ of patients receiving everolimus plus exemestane and $12 \%$ of those receiving exemestane plus placebo reported serious AEs, and discontinuation because of an AE was also higher with everolimus compared with placebo (19 vs. 4\%) [32]. The most frequently reported grade 3/4 AEs in the everolimus arm were stomatitis, anemia, dyspnea, fatigue and hyperglycemia. Among patients receiving neoadjuvant everolimus plus letrozole, $52.9 \%$ required dose interruption or discontinuation, compared with 7.6\% among those receiving letrozole alone [36]. Stomatitis was the most frequent $\mathrm{AE}$ reported in this combination, and hyperglycemia was the most frequent grade $3 / 4$ AE. Three patients developed symptomatic pneumonitis, which resolved in all cases within 15 days of treatment discontinuation [36]. In patients with metastatic breast cancer receiving everolimus (10 $\mathrm{mg} /$ day) in combination with tamoxifen, toxicity-related discontinuations were more frequent in the combination arm (22 vs. $7 \%$ ), with stomatitis, pain and fatigue among the most frequently reported grade 3/4 AEs [35]. For the phase 2 study of everolimus plus fulvestrant, the most common AEs were mucositis, rash, infection and fatigue. However, most of these toxicities were grade 1 , with no grade 4 toxicities observed [37].

HER2-positive metastatic breast cancer studies with everolimus have generally been conducted in heavily pretreated patients who have received $\geq 2$, and in some cases as many as 5, previous treatments [38-40]. In these studies, grade $3 / 4$ neutropenia and leukopenia were the most frequent grade 3/4 AEs and were generally considered attributable to chemotherapy [38-41]. Stomatitis, mucositis and hyperglycemia were the most frequently reported grade 3/4 AEs attributable to everolimus.

\section{Future Directions}

Data from BOLERO- 1 and BOLERO-3 are expected to follow the recent reporting of BOLERO-2. BOLERO-1 and BOLERO- 3 are ongoing phase 3 studies in patients with HER2-positive metastatic breast cancer overexpressing HER2. In BOLERO-1 (NCT00876395) everolimus will be evaluated in combination with paclitaxel and trastuzumab in a first-line setting, and in BOLERO-3 everolimus will be administered in combination with vinorelbine plus trastuzumab in patients who progressed while receiving trastuzumab and who previously received a taxane (NCT01007942).

\section{Other Inhibitors of the mTOR Pathway}

Several other PI3K/mTOR inhibitors are currently being evaluated in early clinical studies in patients with advanced solid tumors (table 3) [45-50]. Deforolimus (ridaforolimus, AP23573, MK-8669) is a nonprodrug analog of rapamycin that selectively inhibits mTOR activity, causing dose-dependent inhibition of cell growth in vitro and antitumor activity in mouse xenograft models [51]. Phase 1 data indicate an acceptable tolerability profile, with fatigue, anorexia, mucositis, nausea and diarrhea among the most commonly reported AEs [52].

NVP-BEZ235 is a dual PI3K/mTOR inhibitor that has shown growth-inhibitory properties in a human breast cancer cell line resistant to tamoxifen [45]. NVP-BEZ235 also blocks vascular endothelial growth factor-mediated cell proliferation and angiogenesis, inhibits microvessel permeability in vitro [53] and may alleviate resistance to ErbB receptor inhibitors [54].

XL765 is a dual PI3K/mTOR inhibitor that blocks PI3K signaling in vitro and shows antitumor activity in xenograft models [55]. A phase 1 study of 34 patients with 
Table 3. Investigational PI3K/mTOR inhibitors [45-50]

\begin{tabular}{|c|c|c|}
\hline Drug & Mechanism & Clinical trial identifier \\
\hline $\begin{array}{l}\text { Deforolimus } \\
\text { (ridaforolimus } \\
\text { AP23573, MK-8669) }\end{array}$ & nonprodrug analog of rapamycin that selectively inhibits mTOR activity & $\begin{array}{l}\text { NCT00736970, NCT01234857, } \\
\text { NCT01220570 }\end{array}$ \\
\hline NVP-BEZ235 & orally available dual PI3K/mTOR inhibitor & $\begin{array}{l}\text { NCT01285466, NCT01248494, } \\
\text { NCT00620594, NCT01482156, } \\
\text { NCT01471847, NCT01495247, } \\
\text { NCT01300962 }\end{array}$ \\
\hline XL765 & dual PI3K/mTOR inhibitor & NCT01082068 \\
\hline GDC-0941 & selective $\mathrm{PI} 3 \mathrm{~K}$ inhibitor & NCT00928330, NCT00960960 \\
\hline GDC-0980 & dual PI3K/mTOR inhibitor & NCT01254526 \\
\hline GSK2126458 & $\begin{array}{l}\text { dual PI3K/mTOR inhibitor that inhibits tumor cell growth and inhibits } \\
\text { PI3K/mTOR signaling in vitro [45] }\end{array}$ & NCT01248858, NCT00972686 \\
\hline AZD8055 & $\begin{array}{l}\text { ATP-competitive mTOR inhibitor selective for all class I PI3K isoforms } \\
\text { that causes significant growth inhibition in a range of human tumor } \\
\text { xenograft models [46] }\end{array}$ & NCT00731263 \\
\hline OSI-027 & $\begin{array}{l}\text { inhibitor of mTORC1 and mTORC2 that inhibits proliferation of cancer cell } \\
\text { lines with activated PI3K/Akt signaling, and antitumor activity in several } \\
\text { human xenograft models [47] }\end{array}$ & NCT00698243 \\
\hline CH5132799 & selective class I PI3K inhibitor [48] & NCT01222546 \\
\hline $\begin{array}{l}\text { PF-05212384 } \\
\text { (PKI-587) }\end{array}$ & $\begin{array}{l}\text { dual PI3K/mTOR inhibitor that inhibits tumor growth in human breast cancer } \\
\text { xenograft models [49] }\end{array}$ & NCT00940498 \\
\hline ZSTK474 & PI3K inhibitor that has shown efficacy in human cancer xenograft models [50] & NCT012800487 \\
\hline
\end{tabular}

mTOR = Mammalian target of rapamycin; PI3K = phosphatidylinositol-3-kinase; ATP = adenosine triphosphate.

advanced solid tumors revealed that a maximum tolerated dose of $50 \mathrm{mg}$ twice daily was sufficient to inhibit PI3K and pERK signaling [56].

GDC-0941, a selective PI3K inhibitor, has demonstrated inhibition of breast cancer xenograft growth. In vitro studies also suggest that both GDC-0941 and the dual PI3K/mTOR inhibitor GDC-0980 exhibit synergistic antitumor activity when added to trastuzumab-DM1 (trastuzumab conjugated to the antimicrotubule agent maytansinoid) in breast cancer xenograft models [57]. Phase 1 data with GDC-0980 and GDC-0941 in patients with advanced solid tumors support further investigation of these agents in larger clinical trials $[58,59]$.

\section{Conclusions}

The PI3K/Akt/mTOR pathway occupies a pivotal role in the cellular processes regulating growth and proliferation. Numerous lines of research suggest this pathway also represents a fundamental regulator of tumor growth and cellular proliferation in cancer cells, and data from in vitro and in vivo models of breast cancer suggest that activation of the mTOR pathway is implicated in the mechanisms that lead to resistance to widely used and effective cancer treatments, including trastuzumab, lapatinib, endocrine therapy and cytotoxic chemotherapies. Preclinical data indicate that mTOR inhibition overcomes resistance to these drugs. If confirmed clinically, these findings would suggest that mTOR inhibitors have the potential to extend the utility of these agents in patients whose disease has become refractory. 
Several inhibitors of the mTOR pathway are under evaluation for potential use in breast cancer. These studies will ultimately determine whether the mechanistic laboratory observations can be translated effectively into a clinical setting. Early observations from phase 1 and phase 2 clinical trials are promising; in particular, the data reported from the randomized phase 2 TAMRAD trial evaluating everolimus plus tamoxifen in hormone receptor-positive metastatic breast cancer patients demonstrated a significant improvement in TTP for the combination compared with tamoxifen alone. Furthermore, the recent phase 3 trial data from BOLERO-2 confirm significant benefit associated with everolimus therapy in patients with advanced hormone receptor-positive disease. With the BOLERO-2 data, the FDA recently approved the use of everolimus for postmenopausal women with advanced hormone receptorpositive, HER2-negative breast cancer in combination with exemestane after failure of treatment with letrozole or anastrozole. Additional confirmation of the efficacy of mTOR inhibitors in breast cancer awaits results from other phase 3 clinical trials, such as the BOLERO-1 and BOLERO-3 studies of everolimus. Several other exciting drug candidates, including dual PI3K and mTOR inhibitors, are now at earlier stages of clinical development. Accumulating clinical experience with the use of these drugs will provide a greater insight into how best to harness their potential.

\section{Acknowledgments}

Supported by funding from Novartis Pharmaceutical Corporation. Dr. LoRusso has received research funding and sponsorship for clinical trials from Novartis. The author wishes to thank Tim Ibbotson, PhD, Matthew Grzywacz, PhD, and ApotheCom (supported by funding from Novartis Pharmaceutical Corporation) for their editorial and technical support in the development of the manuscript.

\section{References}

1 Jemal A, Siegel R, Xu J, Ward E: Cancer statistics, 2010. CA Cancer J Clin 2010;60:277300.

2 National Comprehensive Cancer Network. NCCN Clinical Practice Guidelines in Oncology ${ }^{\mathrm{TM}}$ : Breast Cancer. 2011. http://www. nccn.org/professionals/physician_gls/f_ guidelines.asp. Accessed February 23, 2012.

-3 Germano S, O’Driscoll L: Breast cancer: understanding sensitivity and resistance to chemotherapy and targeted therapies to aid in personalised medicine. Curr Cancer Drug Targets 2009;9:398-418.

$\checkmark 4$ Borders EB, Bivona C, Medina PJ: Mammalian target of rapamycin: biological function and target for novel anticancer agents. Am J Health Syst Pharmacy 2010;67:2095-2106.

5 McAuliffe PF, Meric-Bernstam F, Mills GB, Gonzalez-Angulo AM: Deciphering the role of PI3K/Akt/mTOR pathway in breast cancer biology and pathogenesis. Clin Breast Cancer 2010;10(suppl 3):S59-S65.

6 Sheri A, Martin LA, Johnston S: Targeting endocrine resistance: is there a role for mTOR inhibition? Clin Breast Cancer 2010; 10(suppl 3):S79-S85.

$\checkmark 7$ Yip CK, Murata K, Walz T, Sabatini DM, Kang SA: Structure of the human mTOR complex I and its implications for rapamycin inhibition. Mol Cell 2010;38:768-774.

$>8$ Johnston SR: New strategies in estrogen receptor-positive breast cancer. Clin Cancer Res 2010;16:1979-1987.
-9 Margariti N, Fox SB, Bottini A, Generali D: 'Overcoming breast cancer drug resistance with mTOR inhibitors'. Could it be a myth or a real possibility in the short-term future? Breast Cancer Res Treat 2011;128:599-606.

10 Nagata Y, Lan KH, Zhou X, et al: PTEN activation contributes to tumor inhibition by trastuzumab, and loss of PTEN predicts trastuzumab resistance in patients. Cancer Cell 2004;6:117-127.

11 Junttila TT, Akita RW, Parsons K, et al: Ligand-independent HER2/HER3/PI3K complex is disrupted by trastuzumab and is effectively inhibited by the PI3K inhibitor GDC-0941. Cancer Cell 2009;15:429-440.

12 Berns K, Horlings HM, Hennessy BT, et al: A functional genetic approach identifies the PI3K pathway as a major determinant of trastuzumab resistance in breast cancer. Cancer Cell 2007;12:395-402.

13 Perez-Tenorio G, Stal O: Activation of AKT/ $\mathrm{PKB}$ in breast cancer predicts a worse outcome among endocrine treated patients. $\mathrm{Br} \mathrm{J}$ Cancer 2002;86:540-545.

14 deGraffenried LA, Friedrichs WE, Russell $\mathrm{DH}$, et al: Inhibition of mTOR activity restores tamoxifen response in breast cancer cells with aberrant Akt Activity. Clin Cancer Res 2004;10:8059-8067.

15 Kim EK, Kim HA, Koh JS, et al: Phosphorylated S6K1 is a possible marker for endocrine therapy resistance in hormone receptor-positive breast cancer. Breast Cancer Res Treat 2011;126:93-99.
16 Tokunaga E, Kimura Y, Mashino K, et al: Activation of PI3K/Akt signaling and hormone resistance in breast cancer. Breast Cancer 2006;13:137-144

17 Sadler TM, Gavriil M, Annable T, Frost P, Greenberger LM, Zhang Y. Combination therapy for treating breast cancer using antiestrogen, ERA-923, and the mammalian target of rapamycin inhibitor, temsirolimus. Endocr Relat Cancer 2006;13:863-873.

18 Del Bufalo D, Ciuffreda L, Trisciuoglio D, et al: Antiangiogenic potential of the mammalian target of rapamycin inhibitor temsirolimus. Cancer Res 2006;66:5549-5554.

19 Buckner JC, Forouzesh B, Erlichman C, et al: Phase I, pharmacokinetic study of temsirolimus administered orally to patients with advanced cancer. Invest New Drugs 2010;28: 334-342.

20 Carpenter JT, Roche $\mathrm{H}$, Campone $\mathrm{M}$, et al: Randomized 3-arm, phase 2 study of temsirolimus (CCI-779) in combination with letrozole in postmenopausal women with locally advanced or metastatic breast cancer. Presented at the 2005 ASCO Annual Meeting, May 13-15, 2005, Orlando, Fla., USA.

21 Chow LWC, Sun Y, Jassem J, et al: Phase 3 study of temsirolimus with letrozole or letrozole alone in postmenopausal women with locally advanced or metastatic breast cancer. Presented at the 29th Annual San Antonio Breast Cancer Symposium, December 14-17, 2006, San Antonio, Tex., USA. 
22 Chan S, Scheulen ME, Johnston S, et al: Phase II study of temsirolimus (CCI-779), a novel inhibitor of mTOR, in heavily pretreated patients with locally advanced or metastatic breast cancer. J Clin Oncol 2005;23: 5314-5322.

23 Raymond E, Alexandre J, Faivre S, et al: Safety and pharmacokinetics of escalated doses of weekly intravenous infusion of CCI-779, a novel mTOR inhibitor, in patients with cancer. J Clin Oncol 2004;22:2336-2347.

24 Atkins MB, Hidalgo M, Stadler WM, et al: Randomized phase II study of multiple dose levels of CCI-779, a novel mammalian target of rapamycin kinase inhibitor, in patients with advanced refractory renal cell carcinoma. J Clin Oncol 2004;22:909-918.

-25 Treeck O, Wackwitz B, Haus U, Ortmann O. Effects of a combined treatment with mTOR inhibitor RAD001 and tamoxifen in vitro on growth and apoptosis of human cancer cells. Gynecol Oncol 2006;102:292-299.

-26 Lu CH, Wyszomierski SL, Tseng LM, et al: Preclinical testing of clinically applicable strategies for overcoming trastuzumab resistance caused by PTEN deficiency. Clin Cancer Res 2007; 13:5883-5888.

-27 Beeram M, Tan QT, Tekmal RR, Russell D, Middleton A, deGraffenried LA. Akt-induced endocrine therapy resistance is reversed by inhibition of mTOR signaling. Ann Oncol 2007; 18:1323-1328.

-28 Kirchner GI, Meier-Wiedenbach I, Manns MP. Clinical pharmacokinetics of everolimus. Clin Pharmacokinet 2004;43:83-95.

29 Kovarik JM, Hartmann S, Figueiredo J, et al: Effect of food on everolimus absorption: quantification in healthy subjects and a confirmatory screening in patients with renal transplants. Pharmacotherapy 2002;22:154159.

- 30 Ellard SL, Clemons M, Gelmon KA, et al: Randomized phase II study comparing two schedules of everolimus in patients with recurrent/metastatic breast cancer: NCIC Clinical Trials Group IND.163. J Clin Oncol 2009;27:4536-4541.

31 Maas N, Harbeck N, du Bois A, et al: RADAR: A randomized discontinuation phase II study to determine the efficacy of RAD001 (RAD) in breast cancer (BC) patients (pts) with bone metastases only (GBG41). Presented at the American Society of Clinical Oncology Breast Cancer Symposium, October 8-10, 2009, San Francisco, Calif., USA.

- 32 Baselga J, Campone M, Piccart M, et al: Everolimus in postmenopausal hormone-receptor-positive advanced breast cancer. $\mathrm{N}$ Engl J Med 2012;366:520-529.

33 Hortobagyi GN, Piccart M, Rugo H, et al: Everolimus for postmenopausal women with advanced breast cancer: Updated results of the BOLERO 2 Phase III trial. Presented at the 34th Annual San Antonio Breast Cancer Symposium, December 6-10, 2011, San Antonio, Tex., USA.
34 Piccart M, Noguchi S, Pritchard K I et al: Everolimus for postmenopausal women with advanced breast cancer: updated results of the BOLERO-2 phase III trial. Presented at the American Society of Clinical Oncology Annual Meeting, June 1-5, 2012, Chicago, Ill., USA.

35 Bachelot T, Bourgeir C, Cropet C, et al: Randomized phase II trial of everolimus in combination with tamoxifen in patients with hormone receptor-positive, human epidermal growth factor receptor 2-negative metastatic breast cancer with prior exposure to aromatase inhibitors: a GINECO Study. J Clin Oncol 2012;30:2718-2724.

36 Baselga J, Semiglazov V, van Dam P, et al: Phase II randomized study of neoadjuvant everolimus plus letrozole compared with placebo plus letrozole in patients with estrogen receptor-positive breast cancer. J Clin Oncol 2009;27:2630-2637.

37 Badin F, Romond E, Chambers M, et al: A phase II trial of fulvestrant and RAD001 (everolimus) in patients with metastatic estrogen receptor positive breast cancer after aromatase inhibitor failure; a study in progress. Presented at the 33rd Annual San Antonio Breast Cancer Symposium, December 8-12, 2010, San Antonio, Tex., USA.

38 Jerusalem G, Fasolo A, Dieras V, et al: Phase I trial of oral mTOR inhibitor everolimus in combination with trastuzumab and vinorelbine in pretreated patients with HER2-overexpressing metastatic breast cancer. Breast Cancer Res Treat 2011;125:447-455.

39 Andre F, Campone M, O’Regan R, et al Phase I study of everolimus plus weekly paclitaxel and trastuzumab in patients with metastatic breast cancer pretreated with trastuzumab. J Clin Oncol 2010;28:51105115.

40 Dalenc F, Campone M, Hupperets P, et al: Everolimus in combination with weekly paclitaxel and trastuzumab in patients with HER-2-overexpressing metastatic breast cancer with prior resistance to trastuzumab and taxanes: a multicenter phase II clinical trial. Presented at the 2010 ASCO Annual Meeting, June 4-8, 2010, Chicago, Ill., USA.

-41 Morrow PKH, Wulf GM, Ensor J, et al: Phase I/II trial of everolimus (RAD001) and trastuzumab in patients with trastuzumab-resistant HER2-overexpressing breast cancer. J Clin Oncol 2011;29:3126-3132.

42 Mayer IA, Burris H, Bendel J, et al: A phase Ib trial of RAD001, an mTOR inhibitor, with weekly cisplatin and paclitaxel in patients with HER2-negative metastatic breast cancer. Presented at the 32nd Annual San Antonio Breast Cancer Symposium, December 9-13, 2009, San Antonio, Tex., USA.
43 Gonzalez-Angulo A, Green MC, Murray JL, et al: Open label randomized clinical trial of standard neoadjuvant chemotherapy with paclitaxel followed by FEC (T-FEC) vs. the combination of paclitaxel and RAD001 followed by FEC (TR-FEC) in women with triple receptor-negative breast cancer (TNBC). Presented at the 2011 ASCO Annual Meeting, June 3-7, 2011, Chicago, Ill., USA.

44 Rugo H S, Pritchard K I, Gnant M, et al: Everolimus (EVE) for postmenopausal women with advanced breast cancer (ABC) refractory to letrozole or anastrozole: longterm efficacy and safety results of the BOLERO-2 trial. Presented at the 8th European Breast Cancer Conference, March 21-24, 2012, Vienna, Austria.

-45 Leung E, Kim JE, Rewcastle GW, Finlay GJ, Baguley BC: Comparison of the effects of the $\mathrm{PI} 3 \mathrm{~K} / \mathrm{mTOR}$ inhibitors NVP-BEZ235 and GSK2126458 on tamoxifen-resistant breast cancer cells. Cancer Biol Ther 2011;11:938946.

46 Chresta CM, Davies BR, Hickson I, et al: AZD8055 is a potent, selective, and orally bioavailable ATP-competitive mammalian target of rapamycin kinase inhibitor with in vitro and in vivo antitumor activity. Cancer Res 2010;70:288-298.

47 Bhagwat SV, Gokhale PC, Crew AP, et al: Preclinical characterization of OSI-027, a potent and selective inhibitor of mTORC1 and mTORC2: distinct from rapamycin. Mol Cancer Ther 2011;10:1394-1406.

48 Tanaka H, Yoshida M, Tanimura H, et al: The selective class I PI3K inhibitor CH5132799 targets human cancers harboring oncogenic PIK3CA mutations. Clin Cancer Res 2011;17:3272-3281.

49 Mallon R, Feldberg LR, Lucas J, et al: Antitumor efficacy of PKI-587, a highly potent dual $\mathrm{PI} 3 \mathrm{~K} / \mathrm{mTOR}$ kinase inhibitor. Clin Cancer Res 2011;17:3193-3203.

50 Yaguchi S, Fukui Y, Koshimizu I, et al: Antitumor activity of ZSTK474, a new phosphatidylinositol 3-kinase inhibitor. J Natl Cancer Inst 2006;98:545-556.

- 51 Rivera VM, Squillace RM, Miller D, et al: Ridaforolimus (AP23573; MK-8669), a potent mTOR inhibitor, has broad antitumor activity and can be optimally administered using intermittent dosing regimens. Mol Cancer Ther 2011;10:1059-1071.

52 Hartford CM, Desai AA, Janisch L, et al: A phase I trial to determine the safety, tolerability, and maximum tolerated dose of deforolimus in patients with advanced malignancies. Clin Cancer Res 2009;15:14281434

53 Schnell CR, Stauffer F, Allegrini PR, et al: Effects of the dual phosphatidylinositol 3-kinase/mammalian target of rapamycin inhibitor NVP-BEZ235 on the tumor vasculature: implications for clinical imaging. Cancer Res 2008;68:6598-6607. 
-54 Brunner-Kubath C, Shabbir W, Saferding V, et al: The PI3 kinase/mTOR blocker NVPBEZ235 overrides resistance against irreversible ErbB inhibitors in breast cancer cells. Breast Cancer Res Treat 2011;129:387400.

55 Laird D, on behalf of the Exelixis Research and Development: XL765 targets tumor growth, survival, and angiogenesis in preclinical models by dual inhibition of PI3K and mTOR. Presented at the AACR-NCIEORTC International Conference on Molecular Targets and Cancer Therapeutics: Discovery, Biology, and Clinical Applications, October 22-26, 2007, San Francisco, Calif., USA.
56 LoRusso P, Markman B, Tabernero C, et al: A phase I dose-escalation study of the safety, pharmacokinetics (PK), and pharmacodynamics of XL765, a PI3K/TORC1/TORC2 inhibitor administered orally to patients (pts) with advanced solid tumors. Presented at the 2009 ASCO Annual Meeting, May 29June 3, 2009, Orlando, Fla., USA.

57 Sampath D, Fields C, Prior WW, Parsons K, Friedman LS, Lewis-Phillips GD: Combination therapy of the novel PI3K inhibitor GDC-0941 and dual PI3K/mTOR inhibitor GDC-0980 with trastuzumab-DM1 antibody drug conjugate enhances antitumor activity in preclinical breast cancer models in vitro. Presented at the 33rd Annual San Antonio Breast Cancer Symposium, December 8-12, 2010, San Antonio, Tex., USA.
58 Dolly S, Wagner AJ, Bendell JC, et al: A firstin-human, phase l study to evaluate the dual PI3K/mTOR inhibitor GDC-0980 administered QD in patients with advanced solid tumors or nonHodgkin's lymphoma. Presented at the 2010 ASCO Annual Meeting, June 4-8, 2010, Chicago, Ill., USA.

59 Wagner AJ, Von Hoff DH, LoRusso PM, et al: A first-in-human phase I study to evaluate the pan-PI3K inhibitor GDC-0941 administered QD or BID in patients with advanced solid tumors. Presented at the 2009 ASCO Annual Meeting, May 29-June 3, 2009, Orlando, Fla., USA. 